\title{
Interferência de plantas daninhas no cultivo da melancia
}

\author{
Cleber Daniel de G Maciel1; Juliana P Poletine; Edivaldo D Velini²; Dênis R da S Belisário³; Fábio M \\ Martins $^{3}$; Leandro S Alves ${ }^{3}$ \\ ${ }^{1}$ Escola Superior de Agronomia de Paraguaçu Paulista, C. Postal 88, 18600-000 Paraguaçu Paulista-SP; ²UNESP-FCA, Depto. Prod. \\ Vegetal, C. Postal 237, 18603-970 Botucatu-SP; ${ }^{3}$ Alunos Curso de Agronomia, ESAPP; macielconsultoria@hotmail.com
}

\begin{abstract}
RESUMO
A cultura da melancia é uma atividade explorada regionalmente, sendo uma das mais importantes fontes de renda familiar de pequenos municípios do médio Paranapanema, onde mudanças significativas no processo produtivo são atualmente constatadas, passando de mão-de-obra intensiva para uso de tecnologias promissoras, como é o caso do manejo de plantas daninhas. Um experimento foi conduzido no município de Oscar Bressani (SP), em área de produção comercial, com objetivo de estudar a interferência de plantas daninhas, no cultivo da melancia, na safra 2002/2003. O delineamento experimental utilizado foi de blocos ao acaso com dez tratamentos e quatro repetições, representadas por parcelas com área útil de $18 \mathrm{~m}^{2}$, contendo quatro plantas de melancia e infestação prevalecente das espécies Sida spp, Brachiaria humidicola, Commelina benghalensis e Portulaca oleracea. A infestação das plantas daninhas foi estimada através de amostragens aleatórias das parcelas utilizando-se quadro vazado de ferro com 0,5 m de lado. Os tratamentos constaram de testemunhas capinadas e sem capina e diferentes épocas de controle da infestação, de forma que a cultura foi mantida na presença ou ausência das plantas daninhas até 7; 14; 28; 56 e 63 dias após a sua emergência (DAE). A ocorrência do período inicial de convivência possível maior que o período final estabeleceu o Período Crítico de Prevenção da Interferência do $9^{\circ}$ ao $13^{\circ}$ dias (PCPI= 9-13 DAE). A redução média da produtividade em função da interferência das plantas daninhas durante todo o ciclo da melancia foi de $41,4 \%$. As características diâmetro e espessura da casca dos frutos também foram influenciadas pela convivência com a infestação durante todo o ciclo com decréscimos, de 7,9\% e 23,3\%, respectivamente, em média, ao contrário do comprimento e diâmetro de ramas e do ${ }^{\circ}$ Brix da polpa dos frutos, onde não foram constatadas diferenças significativas.
\end{abstract}

Palavras-chave: Citrullus lanatus, interferência, produtividade.

\begin{abstract}
\section{Weeds interference periods in watermelon crop}

Water melon crop is an agricultural activity explored regionally, representing one of the most important sources of family income in small cities of the Médio Paranapanema, São Paulo State, Brazil, where nowadays, significant changes in the yield process are verified, changing from intensive labor to the use of promising technologies, as weed management, for instance. An experiment was carried out at Oscar Bressane municipal district, São Paulo State, Brazil, to study the weed interference on watermelon cultivation, in 2002/2003. Statistical procedure was based on randomized blocks with ten treatments and four replications, represented by plots with useful area of $18 \mathrm{~m}^{2}$, containing four water melon plants and infestation of Sida spp, Brachiaria humidicola, Commelina benghalensis and Portulaca oleracea species. Weed infestation was estimated through randomized samples from the plots, using an iron drained square with $0,5 \mathrm{~m}$ sides. Treatments consisted of checks with and without hand weeded and different periods of weeds control, so that, crop was sustained in weeds presence or absence up to 7; 14; 28; 56 and 63 days after emergency (DAE). Initial period occurrence of possible coexistence greater than the final period established the Critical Period of Interference Prevention from the $9^{\text {th }}$ to the $13^{\text {th }}$ days (CPIP = 9-13 DAE). The reduction in yield due to the weed interference during all water melon crop cycle was about $41,4 \%$. The diameter and thickness of fruits peel were also influenced by the coexistence with weed infestation during all the crop cycle, with decreases of $7,9 \%$ and $23,3 \%$, respectively, against the characteristics length and diameter of branch and ${ }^{\circ}$ Brix of fruits pulp, when significant differences were not observed.
\end{abstract}

Keywords: Citrullus lanatus, weed plants, production.

\section{(Recebido para publicação em 10 de dezembro de 2006; aceito em 22 de fevereiro de 2008)}

\begin{abstract}
A melancieira (Citrullus lanatus Schard.) é uma cucurbitácea originária da África Tropical, cultivada em todo território nacional e em outros países, sendo caracterizada pelo hábito de crescimento rasteiro e pelo ciclo curto (Miranda et al., 1997; Carvalho, 1999; Filgueira, 2000). Planta de fácil adaptação e de amplo uso medicinal, constituíse em uma das principais hortaliças-fruto produzidas e consumidas no Brasil (Miranda et al., 1997; Leonel et al., 2000).

No estado de São Paulo a melancieira é explorada regionalmente
\end{abstract}

(Blanco et al., 1997), sendo uma das mais importantes fontes de renda familiar em pequenos municípios na região do médio Paranapanema. Desta forma, em virtude da adaptação da cultura à região, mudanças significativas no perfil do processo produtivo são atualmente constatadas, passando de mão-de-obra intensiva para investimento em tecnologias promissoras, como é o caso do manejo de plantas daninhas (Maciel et al., 2003).

A convivência com as plantas daninhas pode comprometer a produção da melancieira tanto quantitativa como qualitativamente, onerar o custo de produção e reduzir o rendimento da lavoura. $\mathrm{O}$ uso de herbicidas não é prática comum entre os produtores de melancia, principalmente pela falta de registro de produtos (Blanco et al., 1997; Rodrigues \& Almeida, 1998; Carvalho, 1999), e ao desinteresse das indústrias agroquímicas em investir em culturas consideradas como de menor valor comercial (Durigan, 1992; Miranda et al., 1997; Maciel et al., 2003).

No setor agrícola, onde a competitividade é maior, há de se racionalizar 
custos, o que, no caso de plantas daninhas, significa o estabelecimento de programas mínimos de controle. Para Constantin (1993), esses programas consideram que a interferência das plantas daninhas não se faz sentir durante todo o ciclo da planta. Portanto, é necessário definir os períodos de controle e de convivência com as plantas daninhas, de forma que a produtividade não seja prejudicada. No entanto, o grau de interferência depende de características ligadas à cultura e ao ambiente, como o solo, clima, e manejo do sistema agrícola, assim como pela duração do período em que a cultura convive com as plantas daninhas (Bleasdale, 1960; Zimdhal, 1980; Altieri, 1981).

Para a cultura da melancia, poucos são os resultados de pesquisa evidenciando os prejuízos causados pelas plantas daninhas para diferentes regiões produtoras do Brasil. A literatura menciona a necessidade de controlar-se a infestação de plantas daninhas desde o início do desenvolvimento das plantas daninhas até o fechamento das ramificações da cultura (Blanco et al., 1997; Motoike et al., 1998; Andrade Júnior, 1998; Filgueira, 2000). Medeiros et al. (2000) e Maciel et al. (2002) relataram reduções de produtividade da melancieira submetida à competição durante todo o ciclo de $95 \%$ e $36 \%$, respectivamente, para infestação mista de plantas daninhas e infestação apenas da espécie Digitaria horizontalis.

Entretanto, há relatos na literatura internacional que indicam a melancia como sendo muito sensível à interferência de plantas daninhas, sendo a intensidade da interferência variável com a espécie e densidade da infestação. Buker et al. (2006) relatou que o período crítico de interferência da infestação de Amaranthus spinosus em melancia foi oito dias maior quando comparada à presença de alta infestação de Digitaria sanguinalis. Segundo Buker (2003), duas e oito plantas de Cyperus esculentus por $\mathrm{m}^{2}$ são capazes de reduzir o rendimento da melancieira em $10 \%$ e $80 \%$, respectivamente. Monks \& Schultheis (1998), avaliando densidades de 250 plantas de Digitaria sanguinalis $\mathrm{m}^{-2}$ concluíram que a redução do rendimento da melancieira pode atingir até 90\%. Wallender \& Talbert (1983) estimaram que apenas 2,67 plantas $\mathrm{m}^{-2}$ de Eleusine indica já seriam capazes de promover redução do rendimento da melancieira. Este trabalho teve o objetivo de identificar o período crítico de prevenção da interferência das plantas daninhas em melancia, na região sudoeste do médio Paranapanema.

\section{MATERIAL E MÉTODOS}

O trabalho foi desenvolvido na safra 2002/2003, em área de produção comercial, anteriormente cultivada com pastagem de Brachiaria humidicola, localizada no município de Oscar Bressane (SP), sudoeste do médio Paranapanema. O solo foi classificado como Latossolo Vermelho-distroférrico (textura arenosa), constituído por 78,0\% de areia; $18,0 \%$ de argila e 4,0\% de silte. A análise química do solo, em amostras de 0 a $20 \mathrm{~cm}$ de profundidade revelou $\mathrm{pH}=5,2\left(\mathrm{CaCl}_{2}\right) ; 16 \mathrm{mmol}_{\mathrm{c}} \mathrm{dm}^{-3}$ de $\mathrm{H}^{+}+\mathrm{Al}^{3+} ; 9,0$ mmol $\mathrm{dm}^{-3}$ de $\mathrm{Ca}^{+2} ; 5,0$ $\mathrm{mmol}_{\mathrm{c}} \mathrm{dm}^{-3+}$ de $\mathrm{Mg}^{\mathrm{c}}$; $5,3 \mathrm{mmol}_{\mathrm{c}} \mathrm{dm}^{-3}$ de $\mathrm{K}^{+} ; 15,0 \mathrm{mg} \mathrm{dm}^{-3}$ de $\mathrm{P} ;$ 7,0 $\mathrm{g} \mathrm{dm}^{-3}$ de MO; SB de 19,0 mmol $\mathrm{dm}^{-3}$; CTC de 36,0 $\mathrm{mmol}_{\mathrm{c}} \mathrm{dm}^{-3}$ e V de 54,0\%.

A semeadura da melancia foi realizada em espaçamento de 1,5x3,0 m, em janeiro de 2002, utilizando-se o híbrido Crimson Tide. A adubação na linha de semeadura previamente sulcada foi constituída de 3 t ha ${ }^{-1}$ de esterco-de-galinha e $800 \mathrm{~kg} \mathrm{ha}^{-1}$ da fórmula comercial 08-28-16. Foram efetuadas três coberturas com a fórmula comercial 2005-20, na dosagem de 50 g planta $^{-1}$, em intervalos de dez dias, após a emergência da cultura. Os demais tratos culturais referentes ao controle de pragas e doenças foram realizados periodicamente pelo produtor, segundo recomendações técnicas para cultura da melancia (Miranda et al., 1997).

O delineamento experimental adotado foi blocos ao acaso, com dez tratamentos e quatro repetições, com unidades experimentais de $40,5 \mathrm{~m}^{2}$ e área útil de $18 \mathrm{~m}^{2}$ (3,0 x 6,0 m), constituída por duas linhas centrais, contendo quatro plantas de melancia. Os tratamentos constaram de testemunhas capinadas e sem capina e da manutenção das plantas de melancia, na presença ou na ausência das plantas daninhas até 7; 14; 28; 56 e 63 dias após a sua emergência (DAE). Foram realizadas capinas manuais, com enxadas em trabalho superficial, evitando-se danos ao sistema radicular e às ramas da melancieira.
As características avaliadas foram comprimento e diâmetro mediano das ramas; diâmetro, espessura da casca e teor de sólidos solúveis totais ( $\left.{ }^{\circ} \mathrm{Brix}\right)$ dos frutos e produtividade. Para estimar o nível de infestação das plantas daninhas nas épocas de avaliação, duas amostragens aleatórias dentro da área útil das parcelas foram realizadas utilizando-se quadro vazado de ferro com $0,5 \mathrm{~m}$ de lado. As espécies de plantas daninhas foram periodicamente identificadas e quantificadas e, posteriormente, acondicionadas em estufa de secagem à temperatura de $65^{\circ} \mathrm{C}$, por um período de três dias, para quantificação da matéria seca.

Para produtividade foi realizada análise de regressão, com objetivo de determinar os períodos de interferência, sendo que para períodos crescentes sem interferência utilizou-se o modelo polinomial cúbico e, para períodos crescentes com interferência, ou seja, sem o controle das plantas daninhas, o modelo de Gompertz modificado.

$$
\begin{gathered}
\mathrm{Y}=\mathrm{a} * \mathrm{X}^{3}-\mathrm{b}^{*} \mathrm{X}^{2}+\mathrm{c}^{*} \mathrm{X}+15263 \\
\text { Modelo Polinomial Cúbico } \\
\mathrm{Y}=\mathrm{d}-\mathrm{e}^{\left[\mathrm{a}-\mathrm{e}^{\left(-\mathrm{b}-\mathrm{c}^{*} \mathrm{X}\right)}\right]} \\
\text { Modelo de Gompertz Modificado }
\end{gathered}
$$

Onde, $\mathrm{Y}=$ produtividade $\left(\mathrm{kg} \mathrm{ha}^{-1}\right)$; $\mathrm{X}=$ número de dias em que a cultura permaneceu na ausência de controle das plantas daninhas (PAI); d= parâmetro adaptado, caracterizado pela produtividade da cultura na ausência total de interferência; a; b; c= valores estimados pelos modelos.

Em ambos os casos, as análises de regressão foram associadas ao cálculo de intervalo de confiança pelo teste " $t$ ", a $10,0 \%$ de probabilidade, de forma semelhante à metodologia descrita por Velini (1989) e Palma (2000), em trabalho de matocompetição com as culturas da soja e milho. Os modelos selecionados foram os que apresentaram os melhores ajustes aos dados originais (Pimentel Gomes, 1987; Cousens, 1988). As demais características foram submetidas à análise de variância pelo teste $\mathrm{F}$ e, as médias, comparadas pelo teste “t”, a 10,0\% de probabilidade.

\section{RESULTADOS E DISCUSSÃO}

As principais espécies de plantas daninhas encontradas na área experi- 
mental foram guanxumas (Sida spp), braquiária (Brachiaria humidicola), trapoeraba (Commelina benghalensis) e beldroega (Portulaca oleracea). As demais espécies, observadas aleatoriamente e em menor freqüência, foram: poaiabranca (Richardia brasiliensis), carrapicho-carneiro (Acanthospermum hispidium), fedegoso (Senna obtusifolia), tiririca (Cyperus rotundus), picão-preto (Bidens pilosa), maria-pretinha (Solanum americanum), desmódio (Desmodium tortuosum) e guizo-de-cascavel (Crotalaria incana). A maioria dessas espécies está associada a alterações microclimáticas proporcionadas pelo manejo das culturas olerícolas (Durigan, 1992). A guanxuma e a trapoeraba apresentaram as maiores densidades populacionais até o final do ciclo da melancia, quando comparadas com a braquiária e beldroega (Tabela 1).

Em relação à produtividade da melancieira (Figura 1 e Tabela 2), observou-se que a convivência com as plantas daninhas nos tratamentos mais infestados, ou seja, na testemunha com plantas daninhas e nos tratamentos em que o controle da infestação foi realizado somente a partir dos 14; 28 e 56 DAE, causou reduções significativas na produtividade da melancieira. Os modelos de Polinomial Cúbico $\left(\mathrm{R}^{2}=0,96\right)$ e Gompertz (modificado) $\left(\mathrm{R}^{2}=0,99\right)$ ajustaram-se com elevada precisão aos dados originais de produtividade (Figura 1). Para períodos com interferência das plantas daninhas, a análise de regressão indicou que a cultura da melancia conviveu com as plantas daninhas até nove dias após a sua emergência (DAE) sem perdas significativas da produtividade, com intervalo limitante de confiança de $22.063 \mathrm{~kg} \mathrm{ha}^{-1}$. Esses resultados sugerem o que o PAI (Período Anterior a Interferência) foi de nove DAE. Para períodos sem interferência, a análise de regressão indicou intervalo limitante de confiança de 22,3 t ha-1, sendo que valores inferiores são significativamente menores. Desta forma, as plantas daninhas que emergirem após treze dias após a emergência das plantas de melancia não afetaram a produtividade, uma vez que o Período Total de Prevenção a Interferência (PTPI) foi de 13 DAE.

Tabela 1. Freqüência da densidade populacional de plantas daninhas e biomassa seca acumulada ao final dos períodos de convivência com a cultura da melancia. (frequency of weeds population density and dry biomass accumulated at the end of coexistence periods with watermelon crop). Paraguaçu Paulista, ESAPP, 2003.

\begin{tabular}{|c|c|c|c|c|c|}
\hline \multirow{2}{*}{ Planta daninha } & \multicolumn{5}{|c|}{ Freqüência (\%) } \\
\hline & $7 \mathrm{DAE}^{\prime 1}$ & $14 \mathrm{DAE}$ & $28 \mathrm{DAE}$ & 53 DAE & 63 DAE \\
\hline Sida spp (guanxuma) & 36 & 22 & 25 & 22 & 18 \\
\hline Commelina benghalensis (trapoeraba) & 24 & 32 & 27 & 19 & 16 \\
\hline Brachiaria humidicola (capim-braquiária) & 22 & 14 & 7 & 6 & 7 \\
\hline Portulaca oleracea (beldroega) & 10 & 17 & 15 & 11 & 8 \\
\hline \multirow{2}{*}{$\begin{array}{l}\text { Biomassa acumulada da parte aérea } \\
\text { das plantas daninhas }\end{array}$} & \multicolumn{5}{|c|}{ g de MS $\mathrm{m}^{-2}$} \\
\hline & 3 & 11 & 81 & 106 & 176 \\
\hline
\end{tabular}

${ }^{1 /} \mathrm{DAE}=$ dias após a emergência. $\left({ }^{1} \mathrm{DAE}=\right.$ Days After Crop Emergency).

Tabela 2. Diâmetro, espessura da casca, teores de sólidos solúveis ( ${ }^{\circ}$ Brix) médio dos frutos e produtividade da melancieira, submetida as diferentes épocas de interferência. (diameter, peel thickness, soluble solids contents ( ${ }^{\circ} \mathrm{Brix}$ ) of the fruits and watermelon crop yield, submitted to different interference periods.) Paraguaçu Paulista, ESAPP, 2003.

\begin{tabular}{|c|c|c|c|c|}
\hline Tratamentos & $\begin{array}{c}\text { Diâmetro } \\
\text { dos frutos } \\
\text { aos }(\mathrm{cm}) 56 \\
\mathrm{DAE}^{\prime 1}\end{array}$ & $\begin{array}{l}\text { Espessura } \\
\text { da casca } \\
\text { dos frutos } \\
(\mathrm{cm}) \text { na } \\
\text { colheita }\end{array}$ & $\begin{array}{l}\text { Teor de } \\
\text { sólido } \\
\text { solúvel } \\
\text { ('Brix) dos } \\
\text { frutos na } \\
\text { colheita }\end{array}$ & $\begin{array}{l}\text { Produtivida- } \\
\text { de }\left(\mathbf{k g ~ h a}^{-1}\right)\end{array}$ \\
\hline $\begin{array}{l}\text { 1. Testemunha "com } \\
\text { interferência" }\end{array}$ & $64,87 \mathrm{~cd}$ & $1,76 \mathrm{a}$ & 10,57 & 14.702 \\
\hline $\begin{array}{l}\text { 2. Testemunha "sem } \\
\text { interferência" }\end{array}$ & $70,00 \mathrm{a}$ & $1,35 \mathrm{~d}$ & 9,87 & 25.086 \\
\hline 3. Limpo a partir dos 7 dias & 70,25 a & $1,55 \mathrm{bc}$ & 10,25 & 23.432 \\
\hline 4. Limpo a partir dos 14 dias & $63,50 \mathrm{~d}$ & $1,47 \mathrm{~cd}$ & 10,10 & 18.001 \\
\hline 5. Limpo a partir dos 28 dias & $65,75 \mathrm{bcd}$ & $1,82 \mathrm{a}$ & 10,10 & 15.621 \\
\hline 6. Limpo a partir dos 56 dias & $64,25 d$ & $1,63 a b c$ & 10,30 & 14.541 \\
\hline 7. Limpo até os 7 dias & $64,75 \mathrm{~cd}$ & $1,45 \mathrm{~cd}$ & 9,75 & 21.244 \\
\hline 8. Limpo até os 14 dias & $68,75 \mathrm{abc}$ & $1,71 \mathrm{ab}$ & 10,07 & 22.146 \\
\hline 9. Limpo até os 28 dias & $69,25 a b$ & $1,45 \mathrm{~cd}$ & 9,32 & 25.827 \\
\hline 10. Limpo até os 56 dias & $70,50 \mathrm{a}$ & $1,56 \mathrm{bc}$ & 9,82 & 25.601 \\
\hline $\mathrm{F}$ & $2,80^{*}$ & $2,91^{*}$ & $0,66 \mathrm{NS}$ & - \\
\hline C.V. $(\%)$ & 4,98 & 11,29 & 8,45 & - \\
\hline DMS (10 \%) & 4,03 & 0,22 & 1,02 & - \\
\hline
\end{tabular}

${ }^{11}$ Dias Após a Emergência da cultura. $\left({ }^{11} \mathrm{DAE}=\right.$ Days After Crop Emergency); *Valores seguidos da mesma letra na coluna não diferem entre si pelo teste de "t” ao nível de $10 \%$ de probabilidade. (*Means followed by same letter within each column are not significantly different from each other (“t” Test, $\mathrm{p}<0,10)$ ).

${ }^{\mathrm{NS}}$ não significativo. ( ${ }^{\mathrm{NS}}$ not significant)

A ocorrência do período inicial de convivência possível (9 DAE) maior que o período final (13 DAE) confere o estabelecimento do Período Crítico de Prevenção da Interferência no intervalo do $9^{\circ}$ ao $13^{\circ}$ dias após a emergência das plantas de melancia (PCPI = 9-13 DAE). Nessa condição, segundo Velini (1997), a recomendação de controle das plantas daninhas deve ser efetiva, utilizando-se tantas vezes quantas forem necessárias as práticas para minimizar o crescimento da infestação nesta fase. Portanto, o produtor teria como opções para o controle de plantas daninhas o uso de capinas e/ ou herbicidas em pós-emergência dentro do intervalo do $9^{\circ}$ ao $13^{\circ}$ dias após a emergência da cultura, assim como herbicidas em pré-emergência e com efeito residual de pelo menos 13 dias após aplicação. Alguns trabalhos disponíveis na literatura internacional desenvolvidos em condições ambientais, híbridos de melancia e, principalmente, 


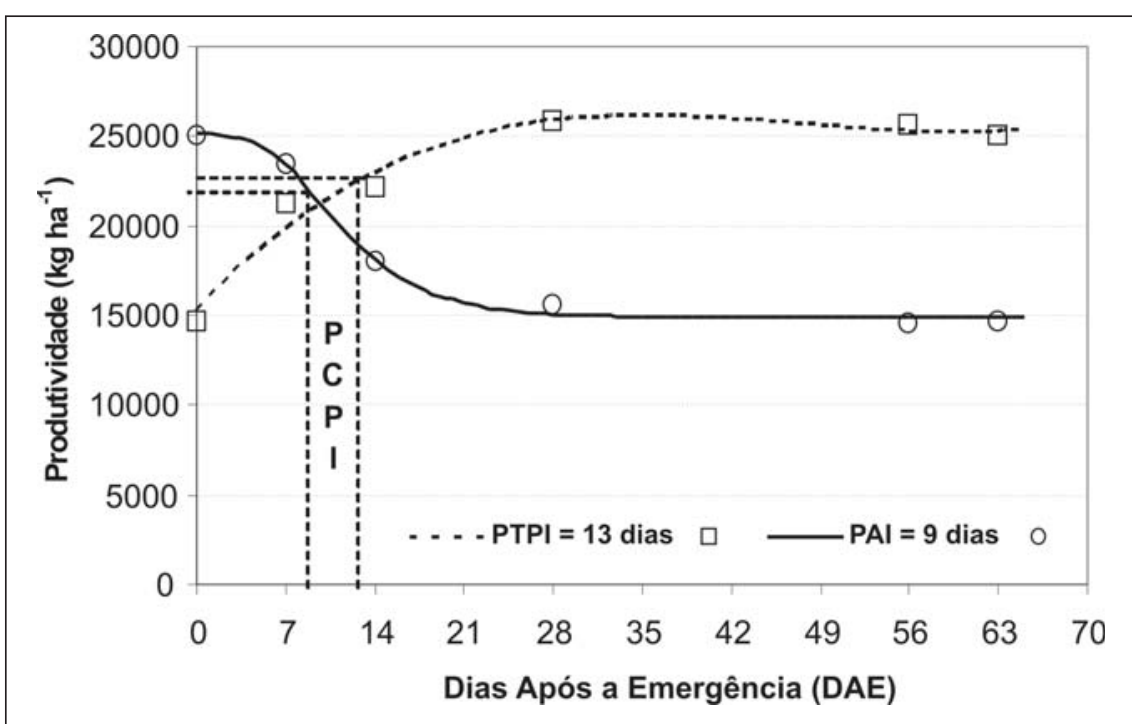

Figura 1. Produtividade de melancia, híbrido Crimson Tide, em função dos períodos de convivência "sem interferência (PTPI)" e "com interferência (PAI)" da infestação de plantas daninhas, resultando no estabelecimento do período crítico de prevenção da interferência (PCPI). (watermelon crop yield, Crimson Tide hybrid, depending on coexistence periods "without interference (PTPI)" and "with interference (PAI)" of weeds infestation, resulting in the establishment of interference prevention critical period (PCPI)). Paraguaçu Paulista, ESAPP, 2003.

infestações de plantas daninhas diferenciadas, relataram o PCPI de 4 a 21 DAE para infestação de Amaranthus hybridus (Terry et al., 1997); de 0 a 42 DAE para melancia transplantada com infestação de Digitaria sanguinalis (Munks \& Schultheis, 1998); de 14 a 28 DAE (Peet, 2005) e de 4 a 21 DAE (Holmes et al., 2006) para infestações mistas.

As características comprimento e diâmetro de ramas, de forma geral, não foram influenciadas em função da presença ou ausência de convivência com a infestação. Diferenças significativas foram encontradas apenas para o comprimento de ramas $42 \mathrm{DAE}$ (dados não apresentados). Ao contrário, o diâmetro dos frutos foi significativamente reduzido em função da interferência das plantas daninhas nos tratamentos mais infestados, de forma semelhante aos resultados de produtividade (Tabela 2). De forma inversa, a espessura da casca dos frutos apresentou-se superior para os maiores períodos de convivência com a infestação, assim como inferiores para as menores épocas de convivência (Tabela 2). Para a característica teor de sólido solúvel ( $\left.{ }^{\circ} \mathrm{Brix}\right)$ nas polpas dos frutos, não foram encontradas diferenças entre os tratamentos.
Em função da falta de informações regionais e do desinteresse da indústria química de herbicidas, além das inúmeras localidades, tipos de materiais e condições ambientais em que a melancia vem sendo cultivada, é de fundamental importância que sejam desenvolvidos novos trabalhos para o conhecimento do período crítico de interferência das plantas daninhas em sistema de semeadura ou plantio de mudas. Com a obtenção de um conjunto mais amplo de informações para a cultura, certamente menos oneroso e mais eficiente será o sucesso do manejo de plantas daninhas, independentemente do processo de manejo adotado na região.

\section{REFERÊNCIAS}

ALTIERI MA. 1981. Weeds may augment biological control of insects. California Agriculture 35: 22-24.

ANDRADE JÚNIOR AS. 1998. A cultura da melancia. Brasília: Embrapa-SPI; Teresina: Embrapa-CPAMN, 86p. (Coleção Plantar, 34).

BLANCO MCSG; GROPPO GA; TESSARIOLI NETO J. 1997. Melancia (Citrullus lanatus Schrad). In: Manual técnico das culturas: Tomo II. 2 ed. Campinas: CATI. p.71-75.

BLEASDALE JKA. Studies on plants competition. 1960. In: HARPER JL. The biology of Weeds. Oxford, Blackwell Scientific Publication. p.113-43.
BUKER III RS; STALL WM; OLSON SM; SHILLING DG. 2003. Season long interference of yellow nutsedge (Cyperus esculentus) with direct-seeded and transplanted watermelon (Citrullus lonatus). Weed technology 17: 751-754.

BUKER III RS. 2006. What You Should Know Before Planning Your Citrus. Weed Management Program. University of Florida, Institute of Food and Agricultural Sciences (UF/IFAS). Disponível em: <http:// edis.ifas.ufl.edu/pdffiles/HS/ HS23500.pdf> Acesso em: 19 out. 2006.

CARVALHO RN. 1999. Cultivo da melancia para a agricultura familiar. Brasília: Embrapa-SPI, $127 \mathrm{p}$.

CONSTANTIN J. 1993. Efeitos de diferentes períodos de controle e convivência da Brachiaria decumbens Stapf com a cultura da cana-de-açúcar (Saccharum spp). 98p. UNESP, Botucatu-SP (Tese mestrado).

COUSENS R. 1988. Misinterpretations of results in weed research through inappropriate use of statistics. Weed Research 28: 281-289.

DURIGAN JC. 1992. Controle de plantas daninhas nas principais culturas olerícolas: umbrelíferas e cucurbitáceas. In: SIMPÓSIO NACIONAL SOBRE MANEJO INTEGRADO DE PLANTAS DANINHAS EM HORTALIÇAS, 1, 1992, Botucatu. Anais... Botucatu: FCA. p.157-86.

FILGUEIRA FAR. 2000. Novo Manual de Olericultura: agrotecnologia moderna na produção e comercialização de hortaliças. Viçosa: UFV. 402p.

LEONEL LAK; ZÁRATE NAH; VIEIRA MC; MARCHETTI ME. 2000. Produtividade de sete genótipos de melancia em Dourados. Horticultura Brasileira 18: 222-224.

HOLMES GJ; MONKS DW; SCHULTHEIS JR; SORENSEN KA, THORNTON AC; TOTH SJ (ed.). 2005. Crop Profile for Watermelons in North Carolina. North Carolina Cooperative Extension Service, North Carolina State University, Raleigh. 12 p. Revised. Disponível em: <http://www.ipmcenters.org/cropprofiles/ docs/NCwatermelons.html>

MACIEL CDG; CONSTANTIN J; GOTO R. 2002. Seletividade e eficiência agronômica de herbicidas no controle de capim-colchão na cultura da melancia. Horticultura Brasileira 20: 474-476.

MACIEL CDG; CONSTANTIN J; GOTO R. 2003. Mato na lavoura. Cultivar Hortaliças e Frutos 20: 24-28.

MEDEIROS RD; MOREIRA MAB; LUZ FJF; OLIVEIRA JUNIOR JOL. 2000. Controle de plantas daninhas na cultura da melancia em Roraima. Horticultura Brasileira 18: 450-451.

MIRANDA FR, RODRIGUES AG; SILVA HR; SILVA WLC; SATURNINO HM; FARIA FHS. 1997. Instruções técnicas sobre a cultura da melancia. Belo Horizonte: EPAMIG. 28p. (EPAMIG, Boletim Técnico, 51)

MONKS DW; SCHULTHEIS JR. 1998. Critical weed-free period for large carbgrass (Digitaria sanguinalis) in transplanted watermelon (Citrullus lanattus). Weed Science 46: 530-532. 
MOTOIKE SY; SALOMÃO LCC; SIQUEIRA DL. 1998. Cultura da melancieira. Viçosa: UFV, Pró-Reitoria de Extensão e Cultura. 25p. (Boletim de extensão, 40)

PALMA V. 2000. Períodos de controle de plantas daninhas na cultura do milho (Zea mays l.). Efeito do número de repetições sobre a precisão experimental. 82p. UNESP, BotucatuSP (Tese doutorado).

PEET M. 2005. Sustainable practices for vegetable production in the south: weed management. Disponível em: <http://www.calls.ncsu.edu/ sustainable/peet/IPM/ weeds/c07weeds.html> Acesso em: 14 set. 2005.
PIMENTEL GOMES F. 1998. A estatística moderna na pesquisa agropecuária. Piracicaba: POTAFOS. 160p.

RODRIGUES BN; ALMEIDAFS. 1998. Guia de Herbicidas. 4 ed. Londrina, PR, Ed. dos autores. 648p.

TERRY ER; STALL WM; SHILLING DG; BEWICK TA; KOSTEWICZ SR. 1997. Smooth amaranth interference with watermelon and muskmelon production. HortScience 32: 630-632.

VELINI ED. 1989. Estudo e desenvolvimento de métodos experimentais e amostrais adaptados à matologia. 250p. UNESP, Jaboticabal-SP (Tese doutorado).
VELINI ED. 1997. Interferências entre plantas daninhas e cultivadas. In: SIMPÓSIO SOBRE HERBICIDAS E PLANTS DANINHAS, 1, Dourados. Resumos... Dourados: Embrapa CPAO. p.29-49. (Embrapa СРAO, Documentos, 13).

WALLENDER CJ; TALBERT RE. 1983. Goosegrass interference with watermelon growth. Proceeding Southern Weed Science Society 36: 158.

ZIMDHAL RL. 1980. Weed crop competition: a review. Corvallis: Oregon State University: International Plant Protection Center. 196p. 